\title{
Single tunneling technique for the resection of lateral spreading adenomas
}

\section{Georgios Mavrogenis, loannis Tsevgas, Panagiotis Kasapidis, Dimitrios Zachariadis}

Mediterraneo Hospital, Athens, Greece

Endoscopic submucosal dissection (ESD) in the colon bears a significant risk of complications due to the fragility of the wall, reduced maneuverability of the endoscope and lack of countertraction. Lately, tunneling methods such as the pocket creation method and the single/multiple tunneling technique have been introduced in an effort to overcome these difficulties [1-3]. Here we describe a single tunneling technique for the resection of a lateral spreading tumor of the transverse colon in a 47-year-old woman with inactive ulcerative colitis (Figs. 1,2). We used the following materials: a $1.5 \mathrm{~mm}$ DualKnife and a CF1 85 coloscope (Olympus), a short hood (Fujifilm), $\mathrm{CO}_{2}$ insufflation and a mixture of hydroxyethyl starch (Voluven, Fresenius Kabi), hyaluronic acid 0.4\% (Sigmavisc, Life Partners Europe) and indigo carmine for submucosal injection. The setting for dissection was Endocut Q, Effect 2 (ERBE VIO3, Tubingen, Germany). The procedure was undertaken under general anesthesia and lasted $2 \mathrm{~h}$. In short, a submucosal tunnel was created underneath the lesion with one proximal and one distal opening. The dissection was then expanded towards the lateral borders and finally the lesion was detached by cutting the lateral margins. This technique allowed a safe dissection of the specimen by a junior ESD-endoscopist, because of the following factors: less dispersion of the submucosal fluid; constant traction and countertraction by pushing the specimen through the tunnel; convenient orientation of the dissection field; good visualization of the vessels; progression of dissection independently of the direction of gravity; and good stability of the endoscope. The patient was hospitalized for $24 \mathrm{~h}$ and had an uneventful recovery. Histology disclosed a $4-\mathrm{cm}$ tubulovillous adenoma with low-grade dysplasia. In conclusion, single tunneling technique may be used for safe and easier resection of lateral spreading tumors. Further studies are needed to establish the various tunneling methods as essential tools for safer colon ESD.

Department of Endoscopy, Mediterraneo Hospital, Athens, Greece

Conflict of Interest: None

Correspondence to: Georgios Mavrogenis, Kavetsou 10, 81100 Mytilene, Greece, Tel.: +30 22510 55557,

e-mail: mavrogenis@gmail.com

Received 28 May 2017; accepted 8 June 2017; published online 30 June 2017

DOI: https://doi.org/10.20524/aog.2017.0172

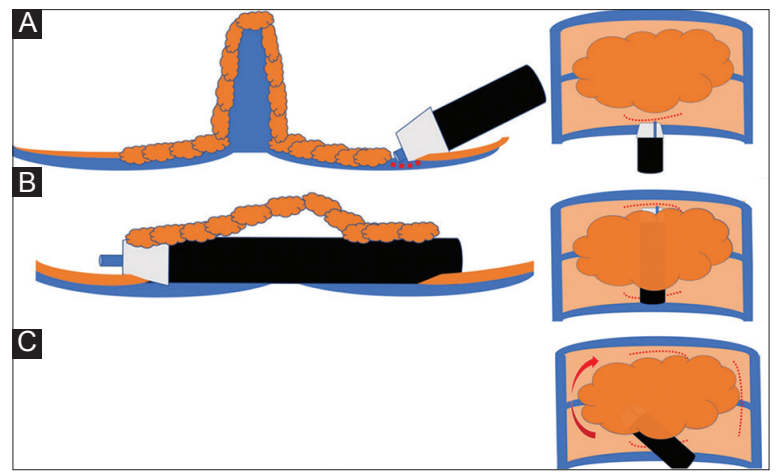

Figure 1 Single tunneling technique. (A) A small incision is made at the anal side of the lesion. (B) An entrance hole is made and then a submucosal tunnel is created towards the oral border of the lesion. Then an opening is created at the end of the tunnel. (C) The dissection is expanded laterally. The final step includes incision of the lateral borders

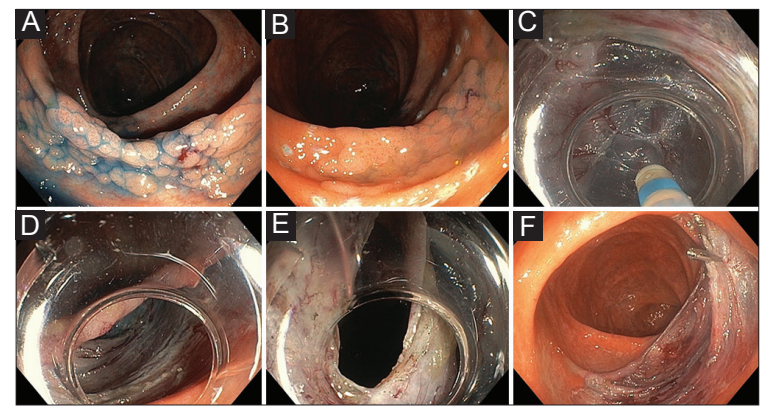

Figure 2 Granular lateral spreading adenoma of the transverse colon expanding on both sides of a semilunar fold. (A) The lateral borders are highlighted with indigo carmine dye spraying. (B) Marking of the periphery. (C, D) Tunneling underneath the lesion. (E) Opening of the tunnel at the oral side of the lesion. (F) Post-endoscopic submucosal dissection defect

\section{References}

1. Sakamoto H, Hayashi Y, Miura Y, et al. Pocket-creation method facilitates endoscopic submucosal dissection of colorectal laterally spreading tumors, non-granular type. Endosc Int Open 2017;5:E123-E129.

2. Aslan F, Kucuk M, Akpinar Z, et al. 1049 Multiple tunneling technique for treatment of rectal circumferential lateral spreading tumor with endoscopic submucosal dissection. Gastrointest Endosc 2016;83:AB197.

3. Aslan F, Kucuk M, Akpinar Z, et al. 737 A comparison of standard endoscopic submucosal dissection technique to the new single tunneling technique in giant laterally spreading tumors. Gastrointest Endosc 2017;85:AB103-AB104. 